\title{
Ausbildung in Landschaftsplanung in der Schweiz
}

\section{Einleitung}

Trotz spürbarer Verlangsamung des wirtschaftlichen und demographischen Wachstums der Schweiz geht die Landschaftsumwandlung und -zerstörung durch zunehmende Intensivierung der Landnutzung durch die Landwirtschaft, die Landbeanspruchung für Siedlungsflächen, Verkehrsflächen, Erholungsräume, Abbau und Deponie und die Nutzung der Luft, des Wassers und des Bodens als Träger für Abfallstoffe und -energien verbunden mit deren chemischen Veränderung ständig weiter.

Die mehrheitlich vorhandene Konsum- und Leistungshaltung der Bevölkerung fördert vorläufig diesen Zerstörungsprozeß, da das sich abzeichnende verstärkte Umweltbewußtsein nur langsam Einfluß auf die Werthaltung gewinnt.

Jede Diskussion über die Ausbildung in Landschaftsplanung in der Schweiz muß deshalb neben der Fachausbildung der Landschafts- und Umwelterziehung auf allen Stufen hohe Priorität beimessen.

Wir beschränken uns nachfolgend auf eine kurze Beschreibung unserer Vorstellungen zur allgemeinen Umwelterziehung, in deren Rahmen unseres Erachtens der Landschaftsschutz integriert sein muß, um im zweiten Teil vertieft auf die eigentlichen Probleme der Fachausbildung einzutreten. Wir sind uns bewußt, daß unsere Analysen und Schlußfolgerungen lückenhaft sind und keinen Anspruch auf Vollständigkeit haben können. Insbesonders unser Postulat auf Schaffung einer Abteilung für Landschaftsplanung an der ETH Zürich soll als Impuls verstanden werden, der dazu dienen könnte, die seit einigen Jahren eingeschlafene Diskussion über die Landschaftsplanungs-Ausbildung auf Hochschulebene wieder etwas anzuregen.

Unter dem Begriff Landschaftsplanung verstehen wir nachfolgend Planungstätigkeiten, die dem Landschaftsschutz i.w.S. dienen. Dieser Begriff umfaßt, gemäß Definition von HUNZIKER, die Bestrebungen des Landschaftsschutzes i.e.S., des Naturschutzes, der Landschaftspflege, des Erholungslandschaftsschutzes und des Heimatschutzes (HUNZIKER 1973).

\section{Die Umwelterziehung auf den verschiedenen Ausbildungsstufen}

Die Anstrengungen zur Weckung und Vertiefung des Landschaftschutzgedankens müssen mit den Bestrebungen, die Umwelterziehung und Umweltbildung in allen Bereichen des Schulwesens zu vertiefen, koordiniert werden.

Deshalb sollen die Anliegen des Landschaftsschutzes im Rahmen eines umfassenden Programms der Umwelterziehung an den Schulen vermehrt geltend gemacht werden. Durch diese Koordination kann eine Vertiefung der Beziehung des Lernenden zur Natur und zur Landschaft erreicht werden.

Nachfolgend einige Gedanken zu den verschiedenen Ausbildungsstufen.

\section{Volksschulen:}

Die Bestrebungen für eine verstärkte Umwelterziehung haben (im Unterschied zur französischsprachigen Schweiz) in der deutschen Schweiz bisher kaum Einzug in die Lehrpläne gehalten. Fragen der Umwelterziehung werden von initiativen Lehrerpersönlichkeiten in den geeigneten Unterrichtsfächern integriert.

Zukünftig sollten die Anliegen des Landschaftsschutzes zusammen mit der generellen Umwelterziehung in die Lehrpläne eingebaut werden. Umwelterziehung sollte nicht als selbständiges Lehrfach, sondern als integrierte Komponente aller geeigneten Lehrfächer behandelt werden. Eine derart umfassende Behandlung des Themas bedingt jedoch eine intensive praktische Ausbildung der Primarlehrer.

\section{Die Vordringlichkeit der Lehrerausbildung und der Lehrerweiterbildung:}

Die Aus- und Weiterbildung von Lehrern ist überaus wichtig, da ihr Einfluß auf die Schüler deren späteres Verhalten prägt. An den Lehrerseminarien muß der Biologie-, der Geographie-, der Geschichts- und Kulturgeschichtsunterricht derart

H.-R. Henz, Raumplaner BSP, und H.-D. Koeppel, dipl. Ing. Landschaftsplaner, Metron AG, Steinackerstraße 7, 5200 Brugg-Windisch. 
gestaltet werden, daß die Beziehung der zukünftigen Lehrer zur Natur- und Kulturlandschaft durch praktische Feldarbeit über Themen des Landschafts-, Heimat- und Umweltschutzes gefördert werden kann.

Schwergewicht der Anstrengungen muß also die Veränderung dieser Unterrichtsfächer sein.

Durch praktische Weiterbildungskurse in der Nähe ihres Wohn- und Schulortes sollten den aktiven Lehrern Themen des Landschaftsschutzes am Beispiel der heimatlichen Landschaft vermittelt werden.

\section{Mittelschulen:}

Auch auf dieser Stufe werden Umweltprobleme vor allem von persönlich interessierten Lehrern behandelt. Es entzieht sich unserer Kenntnis, wie weit der in den Reformvorschlägen «Mittelschule von morgen» der Erziehungsdirektorenkonferenz (1972) vorgeschlagene "pluridisziplinäre Unterricht» gediehen ist, der Fächer aus dem Bereich «natürliche Umwelt» (Physik, Chemie, Biologie, Geowissenschaften) integrieren würde. Ein solches oder ähnliches Projekt würde unseren Vorstellungen der Ausbildung auf der Mittelschulstufe weitgehend entgegenkommen.

\section{Berufsschule, Ingenieurschulen HTL und Försterschulen:}

In den Berufsschulen und den Ingenieurschulen HTL wird die Umwelterziehung nur so weit vermittelt, als dies für die spätere berufliche Tätigkeit direkt notwendig ist. Auch in diesen Schulen sollte durch besondere auf die zukünftigen Berufsbedürfnisse der Schüler ausgerichtete Lehrveranstaltungen den angehenden Berufsleuten gezeigt werden, daß ihre Tätigkeit die natürliche und die bauliche Umwelt beeinflußt und verändert.

Das für die Durchführung solcher Lehrveranstaltungen nötige Grundlagenmaterial ist berufsgruppenbezogen bereitzustellen. Wo eine direkte Verbindung zum Berufsleben nicht möglich ist, sind vermehrt allgemeinbildende Fächer zur Vermittlung des Stoffes zu verwenden.

\section{Umwelterziehung im Bereich der Hochschulen:}

Da Entscheidungen von großer Bedeutung für die Landschaft und die Umwelt oft von Technikern, Juristen, Beamten oder anderen Berufsleuten der privaten Unternehmen oder der öffentlichen Dienste getroffen werden, ist eine Ausbildung von Hochschulabsolventen in Landschafts- und Umweltfragen notwendig. Sie sollen in der Lage sein, Umweltprobleme in ihren eigenen Spezialbereichen zu bewältigen und mit Spezialisten auf verschiedenen Gebieten zusammenarbeiten zu können.
Die Durchsicht der Vorlesungsverzeichnisse der Hochschulen zeigt, daß in der Regel Landschaftsund Umweltprobleme nur sektoral an einzelnen Fachfakultäten gelesen werden. Von Zeit zu Zeit werden im Rahmen einer Ringvorlesung oder eines "collegium generale» fakultäts- und fächerübergreifend Landschafts- und Umweltschutzprobleme behandelt.

An der EPF in Lausanne wird zusätzlich ein Nachdiplomstudium im Bereich «Génie de l'environnement» angeboten. Ähnliche Bestrebungen bestehen in Ansätzen an der ETH in Zürich.

Zur Verbesserung des heutigen Zustandes sollte an den technischen Hochschulen das vorhandene Lehrangebot im Bereich Ökologie/Landschaftsschutz und Denkmalpflege an den verschiedenen Abteilungen ausgebaut werden. Entsprechende Programme sollten durch die interessierten Abteilungen und Institutionen erstellt und durchgeführt werden. Vermehrt sollten Umweltprobleme in allen Lehrgängen integriert und direkt auf den Gegenstand bezogen vermittelt werden.

An den übrigen Hochschulen müssen die vorhandenen Ringvorlesungen zu Kurzlehrgängen für alle Fakultäten und Fachbereiche ausgebaut werden, um einer Vielzahl von Studenten einen Überblick über die gegenwärtigen Probleme des Landschafts-, Heimat- und Umweltschutzes zu geben.

\section{Umwelterziehung und Erwachsenenbildung:}

Das in den vorangegangenen Abschnitten über die Umwelterziehung Gesagte gilt auch für die besonderen Veranstaltungen der Erwachsenenbildung. Daneben haben die privaten Organisationen des Natur- und Heimatschutzes eine wichtige erwachsenenbildende Funktion.

Neben der Vortragstätigkeit und dem vermehrten Einsatz der Massenmedien erfüllen spezielle Zentren, wie das Naturschutzzentrum Aletschwald und das Umweltschutzzentrum des WWF in Zofingen erfolgreich wichtige Aufgaben. Es wäre zu prüfen, ob weitere ähnliche Zentren z. B. für den Heimatschutz gefördert werden könnten.

\section{Die Fachausbildungen im Bereiche des Landschaftsschutzes}

Bestand die Aufgabe des Landschaftsschutzes bis vor ca. 15 Jahren vorwiegend darin, besonders wertvolle Bestandteile der Natur- oder Kulturlandschaft zu bewahren und vor Eingriffen zu schützen, so hat sich die Aufgabenstellung in den letzten Jahren stark erweitert.

Es muß heute davon ausgegangen werden, daß der Mensch den Naturhaushalt dauernd stört. Die Ballungs- und Entleerungstendenzen und damit 


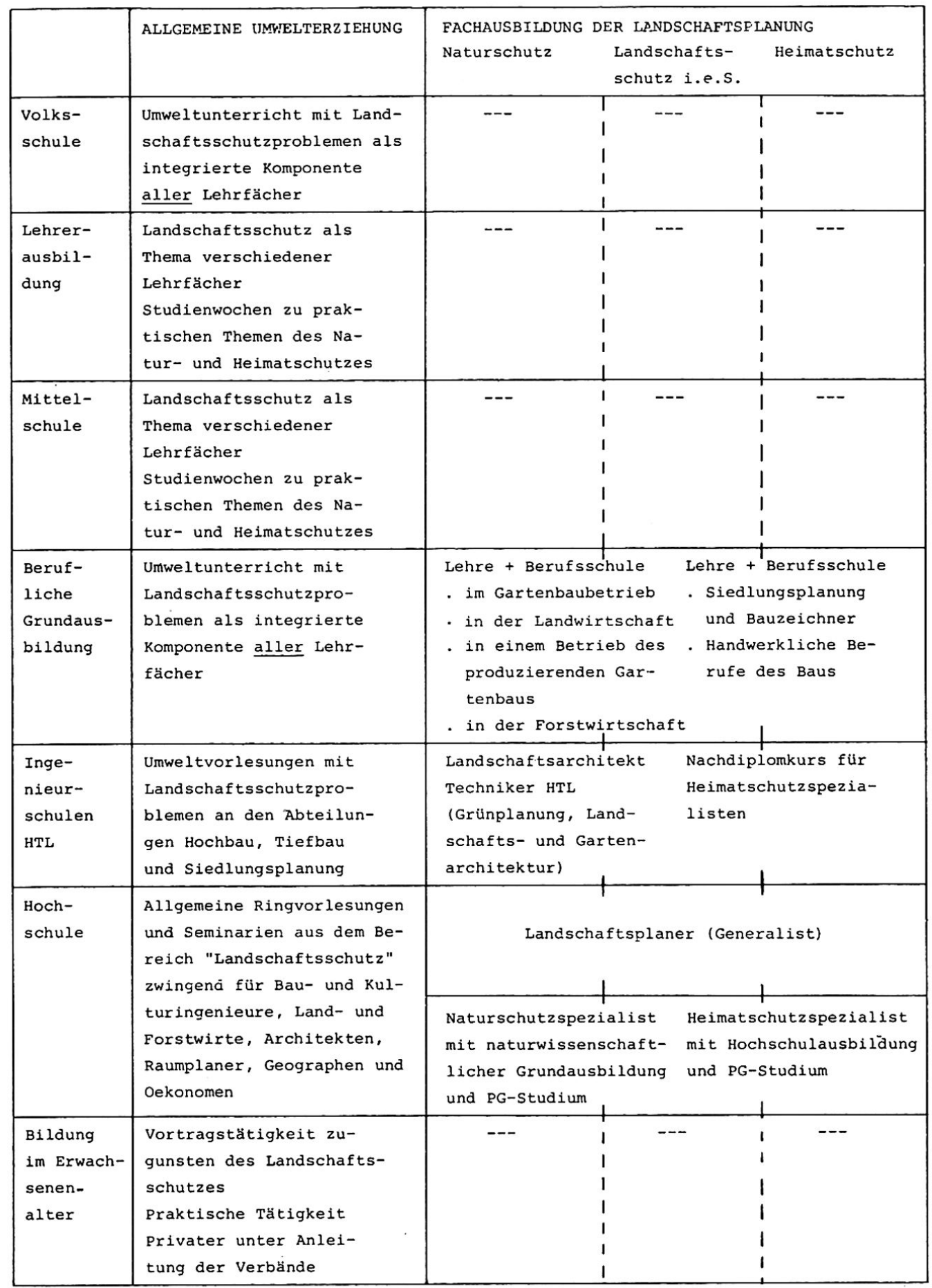

zusammenhängende Nutzungsänderungen fordern die aktive Pflege und Gestaltung der Landschaft (Landschaftspflege). Besonders wertvolle Objekte müssen weiterhin geschützt und gepflegt werden. Darüber hinaus fordern aber heute die gesamte freie Natur und der Siedlungsraum Schutz und Pflege, damit Eingriffe kontrolliert erfolgen und Nutzungsveränderungen mit der kleinstmöglichen Störung des Naturhaushaltes geschehen.

Daraus ergibt sich, daß die Ausbildung der im Landschaftsschutz Tätigen neu überdacht und mit den verwandten Bestrebungen des Umweltschutzes und der Raumplanung koordiniert werden müssen. Das Schwergewicht der Bemühungen muß dabei im Bereiche der Hochschulen liegen, da dort zurzeit die wichtigsten Ausbildungslücken bestehen.

\section{Ausbildung im Bereiche der Hochschule:}

Zurzeit gibt es in der Schweiz, im Gegensatz zu anderen Staaten, keine eigentliche umfassende Ausbildungsmöglichkeit für Landschaftsplaner und 
Naturschützer. Zwar eignen sich verschiedene traditionelle Studienrichtungen für eine Tätigkeit im Interesse des Landschaftsschutzes. Eine Spezialisierung oder Vertiefung dieser Studienrichtung wie Agronomie, Biologie, Botanik, Geographie, Architektur usw. in Richtung Landschaftsschutz ist jedoch nur beschränkt möglich. Einzig die bereits weiter oben erwähnten Nachdiplomstudien in Lausanne und, in Ansätzen in Zürich, eröffnen eine solche Möglichkeit.

Spezialisten, die neben ihrer Grundausbildung mit Teilproblemen des Landschaftsschutzes vertraut gemacht werden, werden an den verschiedenen einschlägigen Hochschulinstituten ausgebildet. In der Regel werden die Teilprobleme jedoch zu einseitig von der Spezialausbildung her gesehen, und es fehlt eine ausreichende Einführung in die komplizierten Zusammenhänge von Landschaft und Umwelt.

\section{Die künftige Ausgestaltung der Ausbildung:}

Aufgrund der geschilderten Veränderungen der praktischen Aufgabenstellung der im Landschaftsschutz tätigen Fachleute sollen in Zukunft folgende Bearbeiter von Landschaftsschutzproblemen ausgebildet werden:

1. Landschaftsplaner als Generalist mit Ausbildungsschwergewicht im Siedlungsbereich oder in der freien Landschaft (Vollstudium).

2. Naturschutzspezialisten mit in der Regel naturwissenschaftlicher Hochschulausbildung und Zusatzstudium in Landschaftsplanung.

3. Heimatschutzspezialisten mit herkömmlicher Hochschulausbildung und Zusatzstudium in Landschaftsplanung.

Der zukünftige Landschaftsplaner als «Generalist» soll über eine sehr breite Ausbildung verfügen. Sie muß ihn befähigen, in der Verwaltung und in der Privatwirtschaft eingesetzt zu werden, um in raumrelevanten Abteilungen Raumansprüche der Öffentlichkeit und Privaten zu koordinieren und den Einsatz von naturwissenschaftlichen Spezialisten zu lenken. Es wäre wünschenswert, wenn dieses Landschaftsplanungsstudium mit den Vertiefungsrichtungen Siedlungsraum und freie Landschaft abgeschlossen werden könnte. Zur Erreichung dieser Ausbildungsziele ist ein Vollstudium nötig.

Landschaftsplaner können den Fachspezialisten nicht ersetzen. Es werden deshalb weiterhin Naturwissenschafter, Spezialisten der Denkmalpflege und Bausachverständige Probleme des Landschaftsund Heimatschutzes bearbeiten. Die Fachspezialisten müssen jedoch in die Grundlagen der Landschaftsplanung eingeführt werden. Es soll deshalb die Möglichkeit geschaffen werden, daß Hochschulabsolventen mit entsprechender Ausbildung ein Nachdiplomstudium für Landschaftsplanung besuchen können.

Aus den Erfahrungen in der BRD und anderen europäischen Ländern lassen sich für die Landschaftsplanung folgende Ausbildungsziele und Schwerpunkte bestimmen.

Ziel der Ausbildung muß es sein, die Studierenden auf Planungsaufgaben in der physischen Umwelt vorzubereiten, die auf mehr oder weniger starke Wechselbeziehungen mit der psychischen Umwelt Rücksicht zu nehmen haben. Die Ausbildung gründet daher auf Natur- und Gesellschaftswissenschaften.

Im naturwissenschaftlichen Bereich müßten Kenntnisse grundlegender Art in Botanik, Pflanzensoziologie und Zoologie verlangt werden mit einer starken Betonung der Ökologie der Pflanzen und Tiere. Notwendig sind Kenntnisse in Klimatologie, Meteorologie, Geographie, Geologie, sowie in Physik und Chemie von Wasser, Boden und Luft. Das Lehrangebot ist so bereitzustellen, daß die Zusammenhänge und Wechselwirkungen von natürlichen Grundlagen und ihrer Nutzung durch den Menschen deutlich werden. Es sind daher auch Grundlagen zu vermitteln über Landwirtschaft, Forstwirtschaft, Verkehrs- und Transportwesen, Siedlungswesen, Abbau von Materialien, Wasser- und Energiegewinnung, Deponie von Materialien und deren Planungsziele, -methoden und -techniken.

Im gesellschaftswissenschaftlichen Bereich sind Kenntnisse über die gesetzlichen Grundlagen des Natur-, Heimat- und Umweltschutzes und über Nationalökonomie zu fordern. In soziologischen Lehrfächern sind Grundlagen über die psychische Umwelt zu vermitteln, die zu ergänzen sind um Kultur- und Kunstgeschichte, Entwurfs- und Gestaltungsfächer. Schließlich müßten Grundkenntnisse der Didaktik gefordert werden.

Im Bereich der Planungswissenschaften wären Lehrangebote in Landschaftsplanung, Naturschutz, Grünplanung, Architektur, Städteplanung und Raumplanung bereitzustellen. Der Ausbildungsschwerpunkt sollte hier auf praktischen Projekten liegen, die geeignet sind, die Arbeitstechniken der Vermessung, des Lebendbaus, des Gartenund Landschaftsbaus und des Hoch- und Tiefbaus zu vermitteln.

\section{Schaffung einer Abteilung für Landschaftsplanung an der ETH Zürich:}

Untersuchen wir das heutige Lehrangebot an den verschiedenen Hochschulen, zeigt sich, daß zurzeit nur an der ETH Zürich die institutionellen und personellen Mittel in den notwendigen Wissenschaftsbereichen vorhanden sind.

Dies führt zum Schluß, daß an der ETH Zürich die Einrichtung eines Zentrums für die Belange des Landschafts- und Heimatschutzes verhältnismäßig einfach möglich wäre. Dies könnte vorerst durch die Einsetzung eines Lehrstuhles geschehen, der später zu einer Abteilung auszubauen wäre. Dieser 
Lehrstuhl für Landschaftsplanung hätte verschiedene Aufgaben zu erfüllen:

- Aufbau und Leitung einer Ausbildung für Landschaftsplanung (Landschaftsplaner als Vollstudium, Natur- und Heimatschutzspezialisten als Nachdiplomstudium)

- Koordination der verschiedenen Wissensbereiche mit dem Ziel, eine systematische Landschaftsforschung aufzubauen

- Bearbeitung von Problemen der Sprache, der Methode und der praktischen Anwendung der Studienergebnisse

- Förderung des Landschaftsverständnisses an den verschiedenen Abteilungen der Hochschule

- Informations- und Dokumentationszentrum für Forschung, Lehre und Praxis.

\section{Ausbildung im Bereiche der Ingenieurschulen HTL:}

Am interkantonalen Technikum Rapperswil ITR und am Centre horticole de Lullier werden heute Landschaftsplaner auf HTL-Stufe ausgebildet. Die Ausbildung wird seit 1976 eidgenössisch anerkannt. Der Aufbau der Ausbildungsrichtung «Grünplanung», Landschafts- und Gartenarchitektur» am ITR und in Lullier entsprechen den Bedürfnissen nach Fachleuten des Landschaftsschutzes. Für die Verwirklichung der Aufgaben des Heimatschutzes sollte auf der Stufe der Höheren Technischen Lehranstalten ein neuer Ausbildungsgang geschaffen werden.

Die Ausbildungsprogramme der übrigen Höheren Technischen Lehranstalten für Hochbau und Siedlungsplanung bieten keine Gewähr, daß die Absolventen dieser Abteilung zur Lösung von Problemen des Heimatschutzes ohne zusätzliche Ausbildung eingesetzt werden können. Es wäre zu prüfen, ob nicht mindestens ein zweisemestriger Nachdiplomkurs an einer HTL-Schule für die Ausbildung von Technikern des Hochbaues, die ihr Wissen im Gebiete des Heimatschutzes und der Denkmalpflege vertiefen möchten, geschaffen werden könnte.

\section{Fachausbildung im Bereiche der Berufsausbildung:}

Für die Belange des Landschaftsschutzes sind die Berufsausbildungen der Gärtner, Förster und Landwirte, für den Heimatschutz die traditionellen handwerklichen Berufe und die eigentlichen Bauberufe von besonderer Bedeutung. Die Ausbildung dieser jungen Berufsleute im Bereiche des Landschaftsschutzes ist bis heute mehr oder weniger zufällig und vom Interesse ihrer Lehrmeister und Berufsschullehrer abhängig.

Die eigentliche Schaffung einer Berufslehre Landschaftspflege ist unseres Erachtens nicht erforderlich. Durch eine Vertiefung der Landschafts- und
Umweltschutzerziehung an den Berufsschulen könnte das Verständnis und die nötige Sachkenntnis verbessert und vertieft werden.

Größere Probleme bietet die Entwicklung in den für den Heimatschutz wichtigen Handwerk- und Bauberufen. Es muß darauf geachtet werden, daß die Handwerker und Baufachleute (Maurer, Steinhauer, Bauzeichner usw.) auch in Zukunft in der Lage sind, unter kundiger Anleitung Denkmäler der Baukunst und des Kunsthandwerkes zu erhalten. Für interessierte Berufsleute sollten entsprechende Kurse und Ausbildungsstellen in Zusammenarbeit mit den verschiedenen Berufsverbänden bereitgestellt werden.

\section{Literatur}

HUNZIKER, TH. (1974): Forderungen des Landschaftsschutzes i.w.S. an die Waldwirtschaft. In Landschaftsschutz und Umweltpflege. Frauenfeld: Huber.

Mittelschule von morgen. Jahrbuch der Schweizer Konferenz der Kantonalen Erziehungsdirektoren. Frauenfeld 1972.

\section{Résumé}

Les sollicitations sans cesse accrues auxquelles doit faire face notre paysage exigent une multiplication des initiatives ayant trait à son aménagement. Toute discussion sur la formation de spécialistes en matière d'aménagement du paysage doit cependant prendre en compte, outre la formation professionnelle proprement dite, les problèmes de sensibilisation à l'environnement et au respect du paysage de l'ensemble de la population.

La protection du paysage, en tant que partie de la sensibilisation à l'environnement, doit être promue à tous les degrés de l'enseignement et de la formation. On attachera une importance particulière à la formation et au perfectionnement des pédagogues. Au degré universitaire, il serait indiqué d'organiser des cours de brève durée donnant à toutes les facultés et disciplines intéressées une vue d'ensemble des problèmes.

On préconise d'instituer, au degré universitaire, un cycle d'études relatives à l'aménagement du paysage (généraliste). Des cours complémentaires à l'intention des spécialistes ayant une autre formation universitaire seraient institués. L'EPF de Zurich se prêterait fort bien à la création d'un centre d'aménagement du paysage. On y procéderait à la formation, la recherche, l'information et la documentation, vu que de nombreux domaines scientifiques connexes se trouvent déjà dans cette école. 